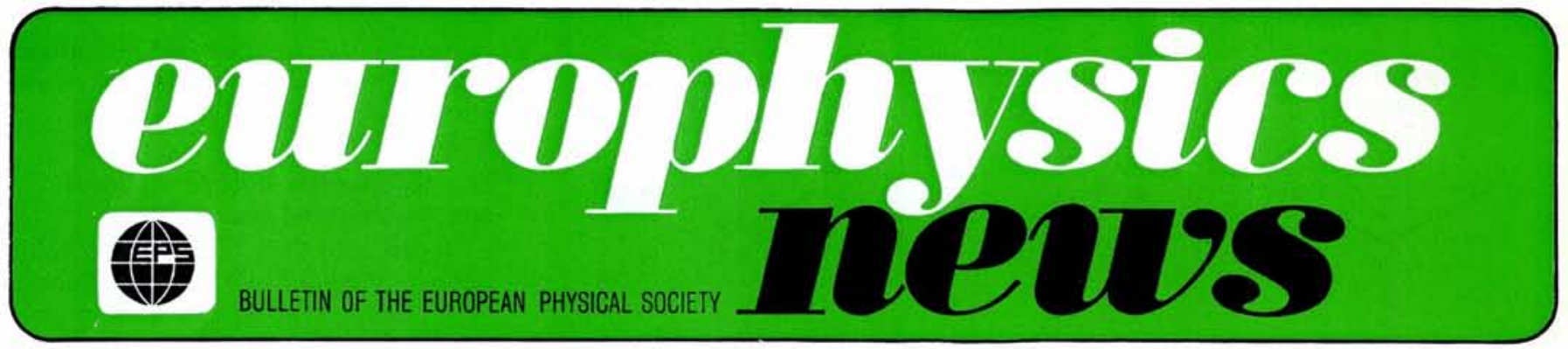

Volume $5 \quad$ Number 4

April 1974

\section{Introduction}

The experimental evidence obtained recently in Gargamelle, a large heavy liquid bubble chamber, at CERN supporting the existence of neutral weak currents may have a capital influence on the present scheme of the particle world. Generally particle physicists assume that everything occuring in our universe is based on four types of interactions between elementary parts of matter called particles. The recent experiments at CERN appear to link two types of basic interactions.

GRAVITATION is the most common interaction. Whilst the weakest of known interactions, it is well known at the macroscopic level. At microscopic level this interaction however produces such small effects that no experiment with individual particles is feasible.

WEAK INTERACTIONS are very much stronger $\left(10^{30}\right)$ than gravitation in the GeV-range. They apparently act as point-like interactions, and give rise to the well known $\beta$-decay of the nucleon. For about 15 years the neutrino, which is only sensitive to weak interactions, has been used as a projectile on nucleons to study the characteristic properties of the weak interaction at different energies.

ELECTROMAGNETIC INTERACTIONS are about $10^{\circ}$ times stronger than weak interactions. They are the interactions known with greatest precision, both experimentally and theoretically. The theory of quantum electrodynamics has been widely tested, to few $\mathrm{ppm}$, and found everywhere in agreement with observations.

STRONG INTERACTIONS are about 100 times stronger than electromagnetic interactions. A great amount of experimental data has been accumulated in the low energy and in the high energy regions. Nevertheless, no global scheme with a sufficient degree of generality to be considered as final theory has come forward.

\section{Towards unification of interactions}

It is a constant aim of many theoreticians to try to find a unified approach for the different types of in-

\title{
Search for Neutral Currents in Gargamelle
}

\section{P. Musset and A. Rousset, CERN}

P. Musset, Maitre de Recherches at the CNRS, and A. Rousset, Professor at the Ecole des Mines, in Paris are presently doing research at CERN, Geneva.

teractions. Let us consider the weak and the electromagnetic ones. The weak interaction theory, first introduced by Fermi and developed in 1957 makes use of a close analogy with electromagnetism by using the concept of "current".

The electromagnetic current in quantum mechanics is carried by a charged particle which remains charged after the interaction, so that the current is said to be neutral. For example in the scattering of an electron on a proton $\left(e^{-} p \rightarrow e^{-} p\right)$ the lepton $\left(e^{-}\right)$has the same charge before and after the interaction. The lagrangian density of the interaction is then given by the product of the current times the electromagnetic potential. By analogy the weak current is carried by a charged (or neutral) particle, which transforms into a neutral (or charged) particle, and gives rise to a charged current. For example a neutrino interacts with a neutron giving an electron and a proton $\left(v+n \rightarrow e^{-}+p\right)$, where the final lepton $\left(e^{-}\right)$has a charge different from that of the incoming lepton (v). A charged current is assumed to interact with another conjugate charged current in the same way as the electromagnetic current is interacting with the electromagnetic field.

In the case of electromagnetism, the interactions may be seen, and are actually mathematically described as due to the exchange of virtual photons (Fig. 1). In the case of weak interactions, it might be that another type of boson is exchanged, a charged one, which has to be heavy, and is really heavier than our present machines are able to produce, i.e. a few $\mathrm{GeV}$ according to the latest experi- ments at National Accelerator Laboratory, USA (NAL). If the two types of interactions are so closely related, why not try to have a unified scheme in which the two kinds of interactions would be considered as different aspects of the same interaction ?

This was attempted at an early stage, before 1960, mainly on the basis of generality : why not a neutral weak current, besides the charged weak current, which permits the unification of weak and electromagnetic interactions. In that case a neutral heavy boson also exists. Then the observable photon and the hypothetical neutral heavy boson are two orthonomal combinations of two basic fields, which are the Yang-Mills fields of the theory. The Yang-Mills theory in which the gauge invariance is related to the existence of such basic fields underlies these ideas.

\section{Gauge theory}

In recent years the interest for these considerations was renewed by attempts of theoreticians to cure the diseases of the weak interaction theory. In the Fermi theory the calculation of cross sections at high energy ( $300 \mathrm{GeV}$ in the centre of mass) violates the strong condition of uni-

\section{Contents}

Search for Neutral Currents in Gargamelle

EPS Council Meeting

European High Pressure Research Group

European Solar Radio Astronomers

Society News

Meetings

1
4
5
6
7
8


Charged weak current

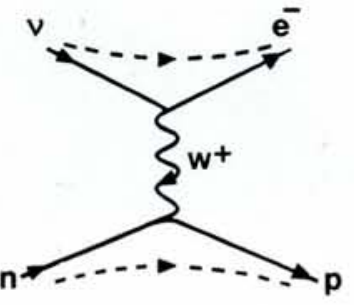

Neutral electr. current

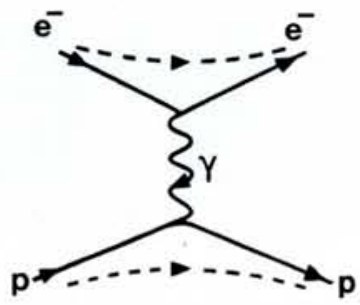

Neutral weak current

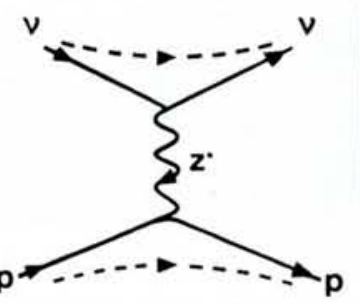

Fig. 1: The three processes $v+n \rightarrow e^{-}+p, e^{-}+p \rightarrow e^{-}+p$, and $v+p \rightarrow v+p$ are believed to proceed via exchange of the intermediate charged boson $W^{+}$, the photon $\vec{\gamma}$, the neutral boson $Z^{\circ}$ respectively.

tarity. The Fermi theory is adequate to describe low-energy interactions but otherwise diverges.

In electromagnetism, a renormalization procedure resolves these problems, using the gauge invariance which applies since the mass of the photon is zero. Again in analogy with electromagnetism, the gauge invariance is introduced, and the bosons which are massless become massive through spontaneous breakdown of symmetry.

In such schemes, the "divergencies" are removed provided new phenomena exist: neutral currents, as in the Weinberg-Salam-Ward model, or heavy leptons, as in the GeorgiGlashow, or Lee-Prentki-Zumino models. Also both neutral currents and heavy leptons could co-exist. Various other models of that sort have been constructed.

\section{Experimental situation before the Gargamelle experiment}

It was actually known for a long time that strangeness-changing inter- actions were not allowed to proceed via neutral currents. That this was not the case was moreover known with a high degree of accurary e.g. the decay induced via a neutral current of the strange meson $\mathrm{K}^{\circ} \rightarrow \mu^{+} \mu^{-}$was known to be $<10^{-8}$ that of the decay $\mathrm{K}^{+} \rightarrow \mu^{+} v$ induced via a charged current.

For the strangeness-conserving neutral current, very little was known before the Gargamelle experiment started. Nevertheless, during the first part of the experiment, first results on processes possibly induced by neutral currents were published. The elastic scattering on electrons

$\bar{v}_{\mathrm{e}}+\mathrm{e}^{-} \rightarrow \bar{v}_{\mathrm{e}}+\mathrm{e}^{-}$was studied at the Savannah nuclear reactor, and the one neutral pion production

$v_{\mu}+$ nucleus $\rightarrow v_{\mu}+\pi^{\circ}+$ nucleus was studied at the Brookhaven accelerator.

For some time, the consistency of the results was believed to be evidence against the possible existence of neutral currents at the level pre-

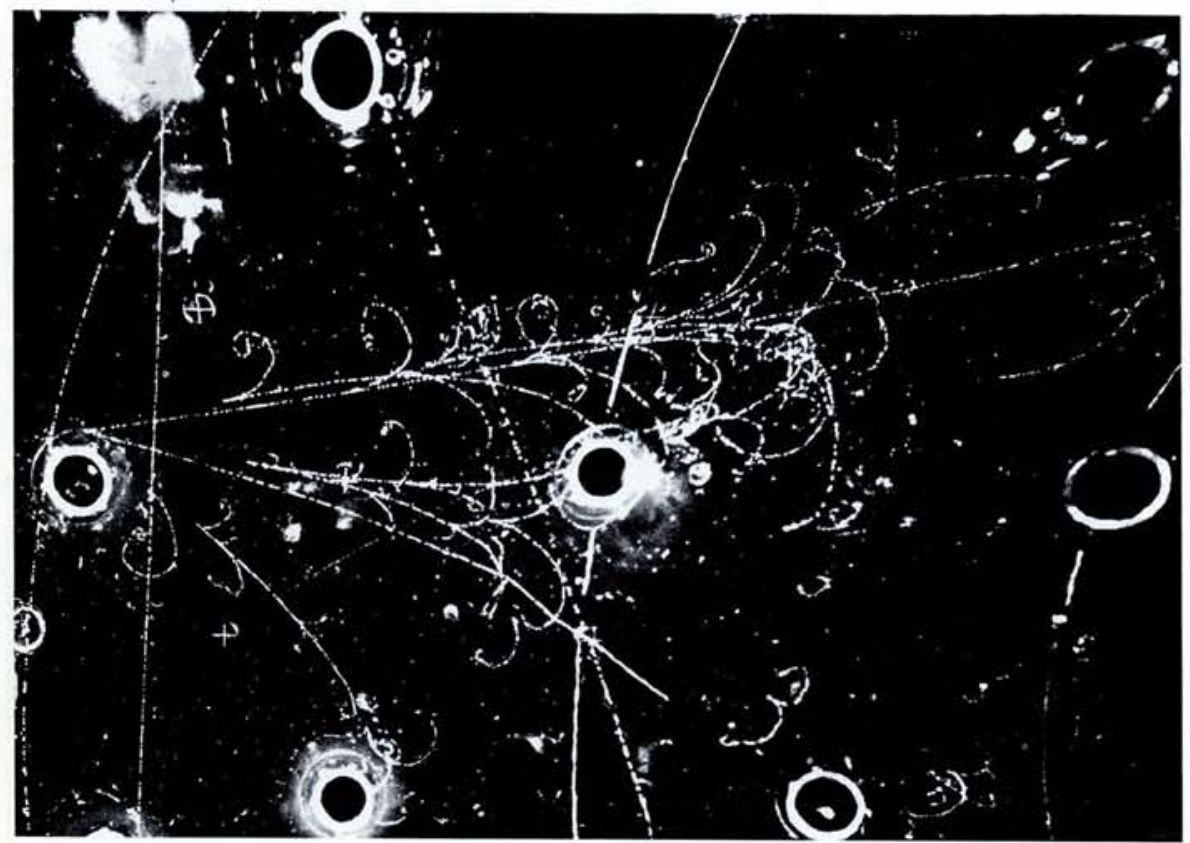

Fig. 2: The non-visible neutrino beam is coming from the left. All particles interact except the muon, giving rise to the longest track.

dicted by the simplest model. Nevertheless, the experimental search was pursued for neutral currents at any level of intensity.

\section{The Gargamelle bubble chamber} and the neutrino beam

Gargamelle is a large heavy liquid bubble chamber built at the Department Saturne at Saclay and in operation since 1971 in the neutrino beam at CERN. The body of the chamber is a cylinder, $4.8 \mathrm{~m}$ long and $1.8 \mathrm{~m}$ in diameter. The mass of the useful sensitive liquids is 10 tons of Freon $\left(\mathrm{CF}_{3} \mathrm{Br}\right)$. The chamber is particularly adapted to the detection of neutrino interactions because the large mass of the detector compensates for the very small cross-section of the neutrinos $\left(10^{-38} \mathrm{~cm}^{2}\right.$ at $\left.1 \mathrm{GeV}\right)$. In addition the large dimensions of the visible volume of Gargamelle permit a good identification of the secondary particles emerging from neutrino interactions: an electron gives a characteristic spiral track in the magnetic field (20000 G), hadrons (meson or nucleon) interact in the liquid with a probability larger than $80 \%$, a neutral pion is recognized by the materialisation of two gammas of the decay $\pi^{\circ} \rightarrow \gamma+\gamma$.

The neutrinos themselves are produced in a special experimental area of the CERN proton synchrotron (PS), the neutrino tunnel. Some $5.10^{12}$ protons of the accelerator are extracted every 2 seconds at the maximum energy of $26 \mathrm{GeV}$ and hit a beryllium target, producing about $10^{13}$ mesons. A large fraction of these mesons decay during their flight along the 70 metres of the tunnel, yielding a muon and a neutrino $\left(\pi^{+} \rightarrow \mu^{+}+v_{\mu}\right)$. An iron shielding, $22 \mathrm{~m}$ thick, stops all the particles except the neutrinos. A flux of about $10^{11}$ neutrinos each 2 seconds is passing through Gargamelle located just behind the shielding. On average, one picture out of ten contains a neutrino interaction.

The analysis of the experiment is done through European collaboration of the Universities of Aachen, Brussels, Milan, Orsay, the University College London, the Ecole Polytechnique Paris, and CERN.

\section{The charged current events} and the neutral current events

Most of the charged current interactions of neutrinos (CC) contain in the final state (Fig. 2) a charged lepton, according to the reaction:

$$
v_{\mu}+\mathrm{N} \rightarrow \mu^{-}+\text {hadrons }
$$

The emerging muon is identified by absence of interactions in the liquid. This reaction is of the charged current type because the electric charge of the lepton is modified in the interaction $\left(v_{\mu} \rightarrow \mu^{-}\right)$. 


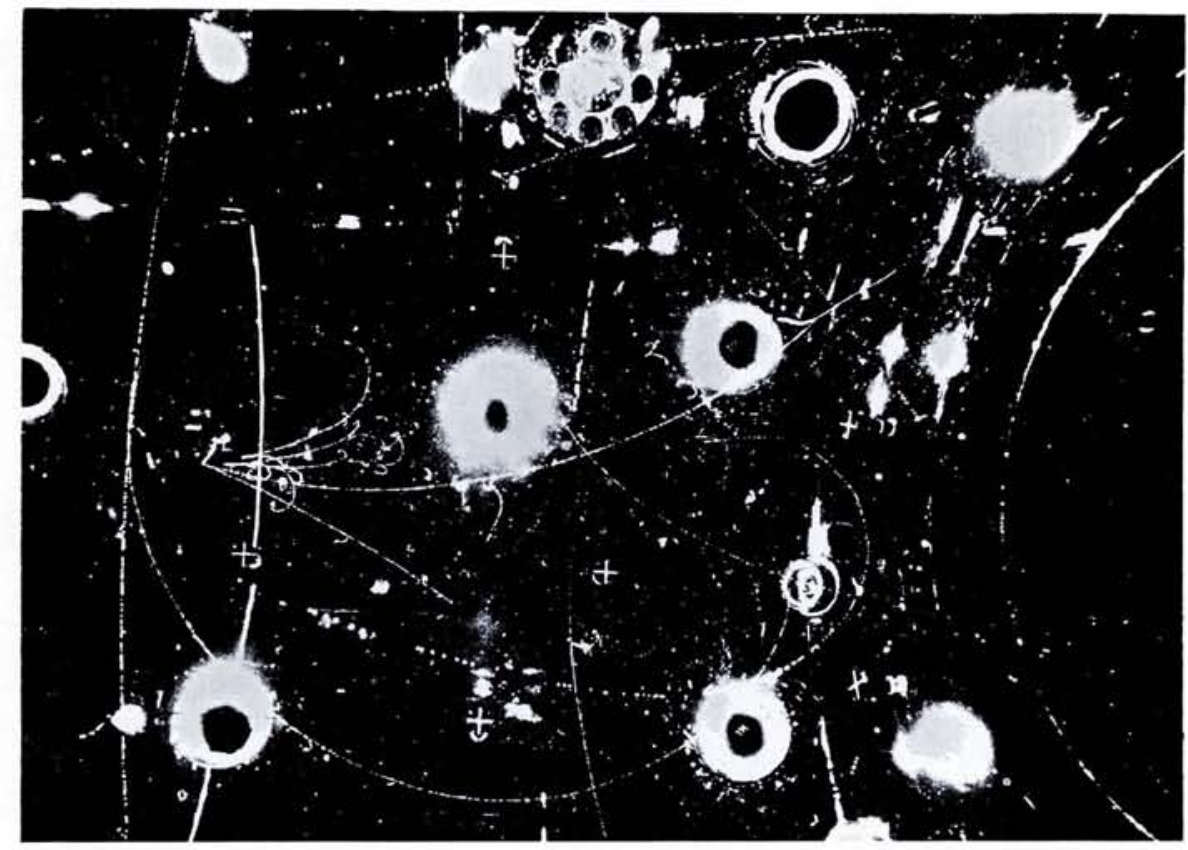

Fig. 3: All particles interact giving stars or $\pi^{\circ}$.

In a neutral current interaction (NC) the electric charge of the lepton is expected to be unchanged after the reaction :

$$
v_{\mu}+\mathrm{N} \rightarrow v_{\mu}+\text { hadrons }
$$

The outgoing neutrino is never detected, and therefore all the visible secondaries in this reaction have to be identified as hadrons. This is possible because interactions with nucleons are unambiguous signatures of hadrons. A search for such events has given 102 and 64 candidates respectively in the neutrino and antineutrino exposures (Fig. 3).

Nevertheless one has to make sure that the signal cannot be simulated by background events. An obvious background could be due to neutrons coming from the PS or coming from neutrino interactions in the material around the chamber. A detailed study of this background shows that no more than $15 \%$ of the signal can be explained that way. Besides, the spatial energetic angular distributions of these 166 events are very similar to the analogue distributions of the charged current events.

\section{The interpretation}

From a strict experimental point of view, one can state that the above is evidence for neutrino-like interactions in which no charged lepton is produced. To interprete the results via neutral current interactions is presently the most plausible interpretation; however because the outgoing neutrino is not detected, this interpretation cannot be considered as unique.

To estimate the cross section of this new process (NC) a comparison was made with the charged current event (CC). A sample of these CC events was selected applying the same criteria to both the complete NC candidate and to the hadronic part of a CC event. The cross sections are in the ratio

$\left(\frac{N C}{\mathrm{CC}}\right)_{v}=0.22 \pm 0.04$ for neutrinos and $\left(\frac{\mathrm{NC}}{\mathrm{CC}}\right)_{v}=0.43 \pm 0.12$ for antineutrinos.

These ratios are relatively large. Also of interest is the fact that the magnitudes of the signal in neutrino and antineutrino exposures are both compatible with the same value of the Weinberg parameter of the most naïve theoretical model.

\section{The leptonic mode}

Another way to prove the possible existence of neutral currents is the search for the purely leptonic reac-

tions: $v_{\mu}+\mathrm{e}^{-} \rightarrow v_{\mu}+\mathrm{e}$

$$
v_{\mu}^{\mu}+e^{-} \rightarrow v_{\mu}^{\mu}+e^{-}
$$

In the simplest theory, the cross section for these processes is expected to be very small $\left(\sim 10^{-41} \mathrm{~cm}^{2}\right.$ at $1 \mathrm{GeV}$ ). Such an event should manifest itself by the appearance of an isolated electron emitted at zero angle along the neutrino beam direction. A special scanning for these events on $5.10^{5}$ pictures with neutrinos and $10^{6}$ pictures with antineutrinos has given respectively 0 and 2 events. A small background is expected from the $v_{e}$ contamination of the beam through the reaction $v_{e}+n \rightarrow e^{-}+p$ in which the proton is not visible. The estimation of this background shows that in the antineutrino exposure of the order of 0.1 event is expected. But in the same number of pictures theory predicts about 3 events of the type $\bar{v}+\mathrm{e}^{-} \rightarrow \bar{v}+\mathrm{e}^{-}$. Therefore the two observed events (Fig. 4) are not likely to be attributed to background, and can also be considered as a serious indication of the existence of the neutral currents.

\section{Conclusion}

In the two reactions studied for the first time evidence is found for the existence of neutral currents. The intensity of the observed phenomena can easily be compared to the simplest theory. This permits the renormalization program and unifies at the same time the scheme of electromagnetic and weak interactions. We are hopeful that these results will be consolidated through future experimental work. If this is the case, then a great impulse will be given to this field of physics in the years to come.

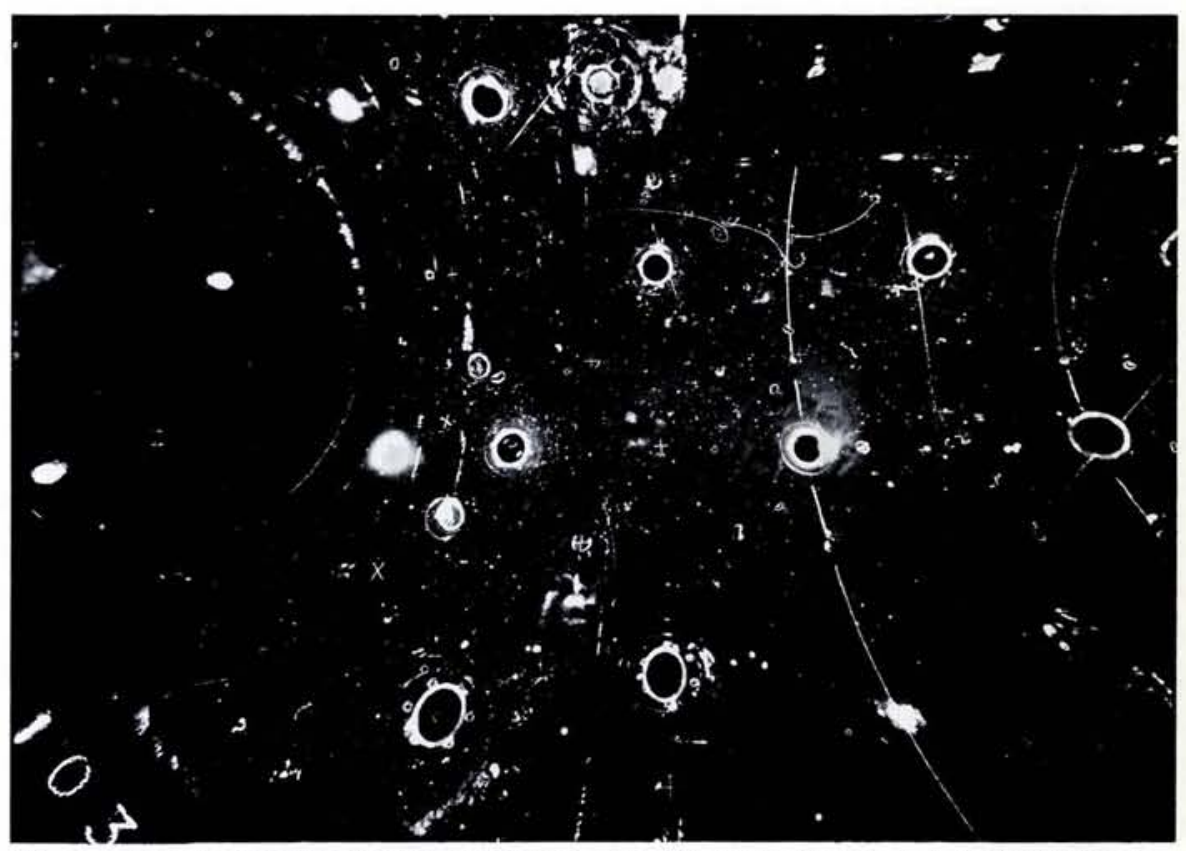

Fig. 4: A very clear electron track is produced at an angle of few degrees from the beam direction. 